CASE REPORT

\title{
A New Palmo-Shoulder Compression Association
}

Hani Sinno*, Teanoosh Zadeh

INTRODUCTION

Carpal tunnel syndrome is a most common peripheral compression neuropathy (1). It is caused by mechanical compression of he median nerve as signs and symptoms are numbness of the lateral three digits and weakness of the thenar muscles due to atrophy (2). Important diagnostic tests include electromyography (EMG) and nerve conduction studies. The gold standard for the surgical treatment is transection of the transverse carpal ligament.

\section{CASE REPORT}

A thirty-eight year old right-handed construction worker presented to the McGill University Health Center Plastic Surgery clinic with complaints of bilateral carpal tunnel syndrome. He had no other relevant past medical history, was not taking any medications, and had no known allergies. On further history, he complained of a ten-year period of slowly progressing symptoms of hand numbness, pain, and paresthesias in the median nerve distri-

On physical examination he demonstrated positive Phalen and Tinel Test bilaterally. He had no obvious thenar eminence wasting and his grip duction studies demonstrated moderate to severe carpal tunnel syndrome bilaterally.$$
\text { A routine open carpal tun }
$$

unnel release was performed on his right hand and the patient had with no complications. Two months lasymtoms scheduled to have the same surgery for his left "To whom correspondence should be addressed:
Dr. Hani Sinno Division of Plastic and Reconstructive Surgery Department of Surgery, McGill Unive
Montreal, QC, Canada H3G 1 A4
Email: hanisisinno@mail.mcgill.ca
DISCUSSION

The median nerve is formed from the medial and lateral cords of the brachial plexus. The arm supplying flexor muscles in the forearm and lateral muscle in the hand, and is responsible for sensation of the lateral part of the palmar surface of the hand. The typical sites of median nerve compression include the carpal tunnel, specifically, beneath Struthers' ligament at the distal humerus, and in the pronator teres muscle. This results in carpal tunnel syndrome, anterior interosseous syndrome, and the pronator syndrome respectively. The median nerve and all its sites of compression have not been shown to cause shoulder weakness and an axilary nerve (C5, $\mathrm{C} 6$ neve root), a branch of the posterior cord of the brachial plexus branch o

The only reported median and axillary nerve combined weakness occur secondarily from proximal compression or defects found in obstetrical injuries, aberrant proximal rib anatomy, and ple. All the described joined median and axillary nerve syndromes are a result of proximal defects. The present case presented with complete resolution of median and axillary nerve weakness after a distal nerve compression surgical procedure. The patient in the present case appears to have an aberrant connection of his median nerve with his axillary nerve or distal compression or the common nerve roots axons that could explain his upstream nerve weakness secondary to the median McGill University. He was a junior editor and then a senior editor for the McGill Journal of Medicine during his medical training at McGill University Medical School. His research interests include reconstructive surgery, and biomedical engineering. the proximal symptoms. neurologic etiologies are found.

\section{REFERENCES} upper extremity. A clinical and
thop Relat Res 1974: 46-67. tunnel surgery. Hand (N Y) 2006: 1: 9-13. jects with carpal tunnel syndrom nerve roots are typically $\mathrm{C} 5-\mathrm{C} 7$. It courses in the to the for that

Hani Sinno (B.Sc., M.Eng., M.D.C.M.) is in his fourth year of plastic surgery residency at

nerve compression in the carpal tunnel. Although it is known that proximal nerve compression such as those seen in TOS can be associated with carpa distal release of the flexor retinaculum can relieve

We have presented a unique case that Lely shows a new peripheral nerve phenonmenon between the axillary and median nerve. This findphysicians, neurologists, plastic surgeons, and orthopedic surgeons who are maneTOS is demontratel syndrome. If an association of ies should be made to rule-out this phen nerve stud We suggest a treatment through sur. carpal tunnel syndrome combined with uniltera choulder abduction weakness in the case where other proximal injuries or abnormal anatomic or

Spinner M, Spencer PS. Nerve compression lesions of the

Vaught MS, Brismee JM, Dedrick GS, Sizer PS, Sawyer Dof disturbances in the thoracic outlet in sub. phenomenon presented in the case report. No results were found. Further searches with heading "carpal tunnel release and "shoulder weakness" " dons" whoulder extension"and "shoulder paralysing the resulted in the same outcome. A search the headings "carpal tunne" and "shoulder (3). The authors have concluded that the likeling f patients with carpal tunnel syndrome having as sociated thocic outet syndrome (TOS) is six astimes higher than control subjects. They have demonstrated that patients with carpal tunnel syndrome may also concomitant proximal nerve entrapment The case presented in this manuscript reveals that a distal release of an entrapped nerve compart ment, in this case the median nerve within the carpal tunnel, has relieved the weakness of a proximal muscle group, the shoulder. 